\title{
More About Human Monocytotropic Ehrlichiosis in Brazil: Serological Evidence of Nine New Cases
}

\author{
Paulo Sérgio Gonçalves da Costa ${ }^{1,2,3}$, \\ Lena Márcia de Carvalho Valle ${ }^{2,3}$, \\ Marco Emilio Brigatte ${ }^{2}$ and Dirceu Bartolomeu Greco ${ }^{1}$
}

\author{
${ }^{1}$ UFMG Medical School, Postgraduate Program in Tropical \\ Medicine, Belo Horizonte; ${ }^{2}$ Monte Sinai Hospital, Juiz de Fora; \\ ${ }^{3}$ João Penido Hospital, FHEMIG, Juiz de Fora, MG, Brazil
}

\begin{abstract}
Human Ehrlichia chaffeensis infections have been reported in North America, Asia and Europe, but only recently have human cases been reported in Brazil. Nine new human cases of $\boldsymbol{E}$. chaffeensis infection diagnosed on a clinical and serological basis are reported. Serological tests were performed with indoor slides prepared with CDC stock DH-82 cells infected with $E$. chaffeensis (Arkansas strain). All but two patients were adults. Seven patients were male and two female. The fever duration varied from 4 to 120 days with a median of 6 days. All patients recalled previous tick attack. IgM was detected in four cases. Influenza like syndrome was the most frequent clinical form affecting five patients. Two patients had fever of unknown origin (FUO), one patient had blood culture-negative endocarditis and one had encephalitis. All patients except one recovered. Two patients were correctly treated. One patient with FUO had AIDS and unexplained pancytopenia. The occurrence of human ehrlichiosis by $E$. chaffeensis remains to be proved in Brazil; the cases reported here highlight the possibility of such disease occurrence in Brazil.
\end{abstract}

Key Words: Monocytotropic ehrlichiosis, Brazil, humans.

"Was man weiss seiht man" [1].
Monocytotropic ehrlichiosis caused by intracellularobligate Gram-negative bacteria Ehrlichia chaffeensis is an emerging human infection transmitted by several tick species, especially Amblyomma spp. [2-5]. This infection has often been reported in North America, Asia and Europe [2-5]; however, serological evidence for infection has also been found in Argentina [6] and Chile [7]. Negative serological evidence for prevalence in humans, but not in dogs, was reported in Brazil by Galvão et al. [8]. However, a high seroprevalence rate was demonstrated in another serosurvey in a cattle-oriented community in Minas Gerais state, Brazil [9].

Ehrlichiosis is a very well known disease among veterinarians in Brazil, where the first published description dates to 1973 [10], and PCR identification has already been obtained from dogs [11]. Only recently, the first human cases of monocytotropic ehrlichiosis were reported on a clinical and serological basis in Brazil by Calic et al. [12]. We report here nine additional symptomatic, serologically diagnosed, monocytotropic ehrlichiosis cases in Brazil.

\section{Material and Methods}

This case-series resulted from a specific protocol to search for rickettsial agents as a cause of fever during a five year period. From 2001 to 2005, 771 febrile patients from five

Received on 11 November 2005; revised 26 January 2006.

Address for correspondence: Dr. LPaulo Sérgio Gonçalves da Costa. Rua Delfim Moreira 181- 902 Juiz de Fora Minas Gerais. Phone numbers: 553232175716,99879514 F. Fax number 553232152653 Email:psgcosta@powerline.com.br.

The Brazilian Journal of Infectious Diseases 2006;10(1):7-10. C 2006 by The Brazilian Journal of Infectious Diseases and Contexto Publishing. All rights reserved. urban medical centers in Juiz de Fora, Minas Gerais, Brazil were systematically screened for rickettsial infections. There were 290 AIDS patients and 481 non-AIDS patients. They were assigned to specific syndromes according to their clinical and laboratory pictures. The serological tests for rickettsial infections were performed by microimmunofluorescence technique (IFA), as described elsewhere [13] using "indoor" slides made with antigens from the Viral and Rickettsial Branch of CDC, Atlanta, USA: Ehrlichia chaffeensis (DH82 infected cells- Arkansas strain), Coxiella burnetii (Nine Mile strain), Rickettsia typhi (Wilmington strain), Rickettsia rickettsii (Sheila Smith strain), Bartonella henselae (Houston strain -Vero cells) and Bartonella quintana (Vero cells).

Fluorescein-conjugated goat anti-human total (IgT), IgG and IgM (Biomérieux) were used for antibody detection.

Sera were initially screened at a dilution of 1:64 PBS in 5\% skim milk and those that were positive were subsequently titered to the endpoint. In the IgM tests, the sera were analyzed with a 1:16 titer. The slide readings were blind and a florescence of at least ++ was considered for positivity.

A search for morulae using Giemsa stain was additionally performed.

Other tests, including chest X-rays, blood, cerebral spinal fluid (CSF) and urine cultures, as well as many serological tests, including those for toxoplasmosis, leptospirosis, cytomegalovirus (CMV), Epstein-Barr virus (EBV), Mycoplasma pneumonia, Chlamydia pneumoniae, Widal, Brucellosis and dengue fever were performed in commercial laboratories.

Anaplasma phagocytophilum antibodies were tested in some (five) cases. Rheumatoid factor absorption was not performed, but most of the samples were directly checked for the presence of rheumatoid factors. The samples were also 
tested for antinuclear antibodies (ANA), because of the cellular substrate nature of the E, chaffeensis antigens.

Diagnostic criteria for monocytotropic ehrlichiosis according to Consensus approach for ehrlichiosis task force-(CAFE) [3,5]). For diagnostic definition, we used the currently accepted criteria, which include compatible clinical (fever, headache and myalgia = influenza like syndrome) and epidemiological (tick attack) findings, plus the following: a) Single reciprocal $\operatorname{IgG}$ titer $\geq 1: 64=$ Possible; b) Single reciprocal IgG titer $\geq 1: 256$ and/or IgM titer $\geq 1: 20$ with or without morulae detection = Probable; $c$ ) Seroconvertion negative-positive or an over four-fold increase in IgG antibody titer $=$ Confirmed and d) Isolation by culture or identification by PCR $=$ Definite. These latter methods were not available in our study.

\section{Results}

Seven patients were male and two were female.

All but two patients were adults: 7 year-old and 15 yearold boys. The age ranged from 7 to 57 years, with a median of 34 years.

The fever duration varied from 4 to 120 days, with a median of 6 days.

All patients recalled a previous tick attack.

A second sample was obtained from four patients in whom seroconversion was detected. Three patients had serological findings compatible with confirmed cases, two with probable cases and four with possible cases.

IgM was detected in four cases.

Three samples had low antibody titers for other rickettsial agents: two were seropositive for Bartonella spp., and the other was seropositive for both Bartonella spp. and R. typhi. One case was positive for rheumatoid factor, but did not show cross-reactivity to any other rickettsial agent. Influenza-like syndrome was the most frequent clinical form, affecting five patients, and cutaneous rash was observed in five cases, four of which with influenza like syndrome.

Two patients had fever of undetermined origin (FUO), one patient had blood culture-negative endocarditis and one, encephalitis. The encephalitis case was preceded by influenzalike syndrome.

The endocarditis patient had mitral valve vegetation with severe structural destruction, which eventually required valve replacement. The patient with encephalitis had both CSF and brain magnetic resonance imaging (MRI) without abnormalities. All but one patient recovered and so the lethality rate in this series was one in nine.

Leukocyte count ranged from 1,200 to $10,800 / \mu \mathrm{L}$, with a median of 9,100, and the platelet counts ranged from 12,000 to $366,000 / \mu \mathrm{L}$, with a median of 101,000 . Aspartate amino transferase (AST) and alanine amino transferase (ALT) levels, available in just seven cases, ranged from 6 and 9, to 75 and 86
$\mathrm{U}$, respectively, with respective medians of 42 and 39 . No morulae were seen in any case, and ANA were negative in all cases.

Two patients were correctly treated with doxycyclin, with fast recovery, and one patient was partially treated with chloramphenicol. Another patient was "accidentally treated"; this was an AIDS patient with FUO and unexplained pancytopenia who empirically received rifampin for a supposed atypical mycobacteria infection. An occurrence rate of $1.7 \%$ among non-AIDS (8 out 481 patients) and of $0.34 \%$ among AIDS ( 1 out 290 patients) was detected. Overall, $1.2 \%$ (9 out 771 patients) had monocytotropic ehrlichiosis as a possible cause of febrile syndromes. The most relevant individual aspects of the reported cases are shown in Table 1.

\section{Discussion}

The occurrence of human ehrlichiosis due to E. chaffeensis or any other Ehrlichia species remains to be proved by bacterium isolation from humans in Brazil. However, human clinical cases with compatible serological evidence have already been reported [12].

A prevalence rate over $10 \%$ was found in a serosurvey carried out in a nearby county, $10 \mathrm{~km}$ from Juiz de Fora, suggesting the presence of such bacteria or antigenically close ones in that particular region [3].

Influenza-like syndrome is known to be the most frequent clinical form of monocytotropic ehrlichiosis [2-5], as we also observed. The clinical course is usually benign and complete recovery is the rule $[2,3,5]$. Two patients were correctly treated with tetracycline (doxycycline), considered the drug of choice for human ehrlichiosis; both recovered rapidly. However, immunocompromised patients can evolve to a bad downhill course [14]. The only fatality found in our series affected a teenager who was immunosuppressed by corticosteroid use and developed severe encephalitis. Central nervous system involvement has been described in monocytotropic ehrlichiosis in the form of meningitis or encephalitis, affecting both immunocompetent and immunosuppressed individuals $[15,16]$. This patient undertook a short course of chloramphenicol therapy, during which he did not show any improvement. This drug is considered non-effective against $E$. chaffeensis [3], but this issue remains somewhat disputable [5].

FUO was the clinical form of two cases, and this feature has been associated with some monocytotropic ehrlichiosis cases [17]. One of the FUO cases affected a patient with AIDS; patients with such illness can suffer severer forms of $E$. chaffeensis infections [14,18], but overall the frequency is considered quite low among HIV-infected patients, even in endemic areas [18]. However, seronegative cases can occur in AIDS patients, thus making serological tests very limited for such patients $[3,18]$. The patient with AIDS received rifampin, considered along with quinolones as an alternative, though less effective way, of treating such infections [2,5]. 


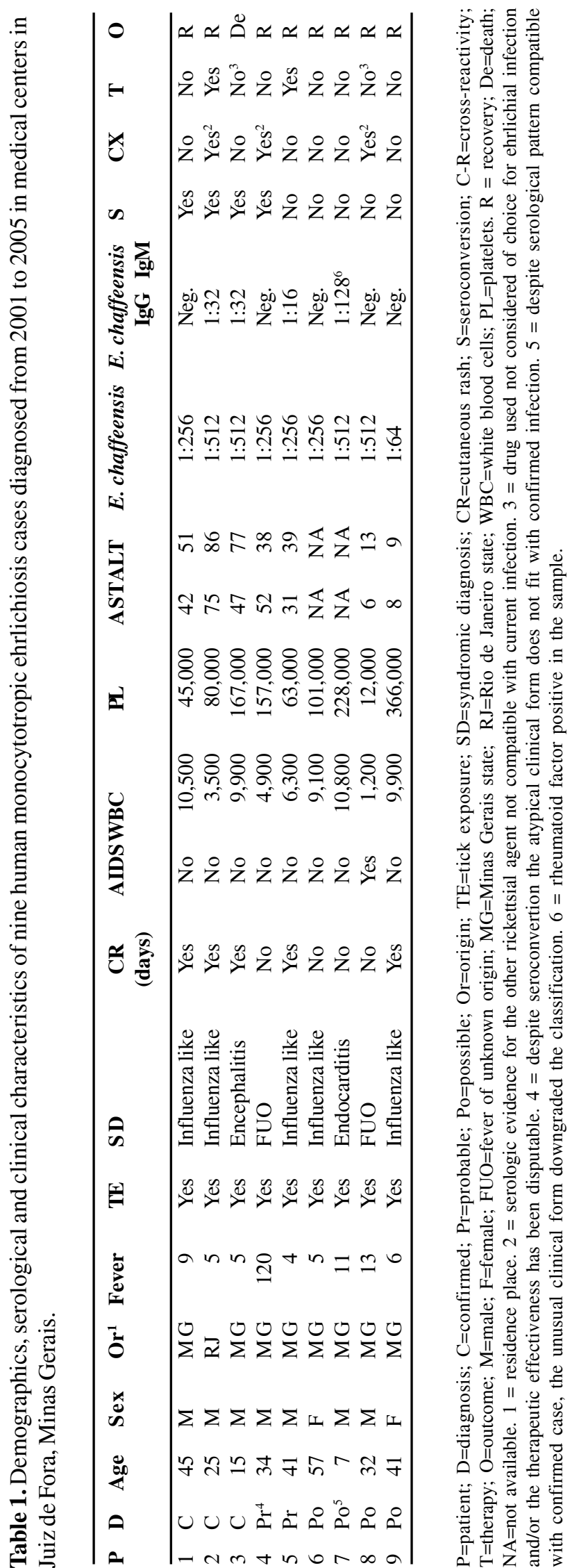

One case was associated with blood-culture-negative endocarditis. Rickettsial agents, especially $C$. burnetii and Bartonella spp. [19], have been described as causative agents of endocarditis, but E. chaffeensis endocarditis has not been reported, although it is considered quite possible [20].

Low platelet counts and a slight increase in AST and ALT, as observed in several cases of this series, have been frequently associated with monocytotropic ehrlichiosis. Together with moderate leukopenia, only detected in a few of the cases in our series, these laboratorial aspects resemble dengue fever but are considered unspecific [2-5].

All these cases had epidemiological and serologic findings compatible with moncytotropic ehrlichiosis [3]. All patients recalled previous tick attack, and this detail has been the rule in human ehrlichiosis [2-5]. From a practical standpoint, diagnosis of human ehrlichiosis relies on serological tests for antibody detection [2-5,21], given the cumbersome nature of culture methods and the very expensive high-tech PCR identification techniques [2-5]. All the patients had positive serological tests for $E$. chaffeensis, but one important limitation of serological diagnosis of ehrlichiosis, as well as that of other rickettsial infections, has been cross-reactivity. The main known crossreactivity in ehrlichiosis has been between $E$. chaffeensis and $A$. phagocytophilum [3,5], but we did not find such cross-reactivity. The cross-reactivity between Bartonella spp. and Ehrlichia spp. that we found in some patients has already been reported [21], as well as between Ehrlichia spp. and R. typhi [9]. The presence of rheumatoid factor has been allegedly responsible for some crossreactive or false positive tests [22], but the only case in our series that had such positivity had no cross-reactivity at all.

\section{Acknowledgments}

We are thankful to Drs. William L. Nicholson, Gregory Dash, James G. Olson, James E. Childs and Russell Regnery from the Viral and Rickettsial branch of CDC Atlanta GA USA for providing the rickettsial antigens. We also thank Dr. Simone B. Calic from FUNED MG, for testing A. phagocytophilum antibodies in some samples.

\section{References}

1. Gonçalves A.J.R., Pinto A.M.M., Melo J.C.P., et al. Febre maculosa brasileira. Considerações relativas a dois casos da cidade do Rio de Janeiro. Jornal Brasileiro de Medicina JBM 1981;41:55-7.

2. CDC. Human ehrlichiosis in the United States. http:// www.cdc.gov/ ncidod/ dvrd/ ehrlichia/Index.htm . Accessed June 5,2004.

3. Paddock C.D., Childs J.E. Ehrlichia chaffeensis: a prototypical emerging pathogen. Clin Microbiol Rev 2003; 16:37-64.

4. Olano J.P., Masters E., Hogrefe W., Walker D.H. Human monocytotropic ehrlichiosis, Missouri. Emerg Infect Dis 2003;9:1579-86.

5. Olano J.P., Walker D.H. Human ehrlichioses. Med Clin North Am 2002;86:375-92. 
6. Ripoll C.M., Remondegui C.E., Ordonez G., et al. Evidence of rickettsial spotted fever and ehrlichial infections in a subtropical territory of Jujuy, Argentina. Am J Trop Med Hyg 1999;61:350-4.

7. Lopez D.J., Rivera S.M., Concha G.J.C., et al. Ehrlichiosis humana en Chile: evidencia serológica. Revista Medica de Chile 2003; 131:67-70.

8. Galvão M.A.M., Lamounier J.A., Bonomo E., et al. Rickettsioses emergentes e reemergentes numa região endêmica do Estado de Minas Gerais, Brasil. Cad Saúde Pública 2002;18:1593-7.

9. Costa, P.S.G. Evidências sorológicas de infecções por Rickettsia rickettsii, Rickettsia typhi, Coxiella burnetii, Bartonella quintana, Bartonella henselae e Ehrlichia chaffeensis em indivíduos sadios e pacientes febris com e sem AIDS da região de Juiz de Fora, Minas Gerais. Ph.D. thesis. Departamento de Medicina Tropical e Infectologia, UFMG, Belo Horizonte Minas Gerais 2004.

10. Moreira S.M., Bastos C.V., Araújo R.B., et al. Retrospective study (1998-2001) on canine ehrlichiosis in Belo Horizonte, MG, Brazil. Arquivos. Brasileiros de Medicina Veterinária e Zootecnia 2003;55:141-7.

11. Dagnone A.S., de Morais H.S., Vidotto M.C., et al. Ehrlichiosis in anemic, thrombocytopenic, or tick-infested dogs from a hospital population in south Brazil. Vet Parasitol 2003; 117:285-90.

12. Calic S.B., Galvão M.A.M., Bacellar F., et al. Human ehrlichiosis in Brazil: first suspected cases. Braz J Infect Dis 2004;8:259-62.

13. Philip R.N., Casper E.A., Ormsbee R.A., et al. Microimmunofluorescence test for the serological study of Rocky Mountain spotted fever and typhus. J Clin Microbiol 1976;3:51-61.
14. Safdar N., Love R.B., Maki D.G. Severe Ehrlichia chaffeensis infection in a lung transplant recipient: a review of ehrlichiosis in the immunocompromised patient. Emerg Infect Dis 2002;8:320-3.

15. Grant A.C., Hunter S., Partin W.C. A case of acute monocytic ehrlichiosis with prominent neurologic signs. Neurology 1997;48:1620-3.

16. Berry D.S., Miller R.S., Hooke J.A., et al. Ehrlichial meningitis with cerebrospinal fluid morulae. Pediatr Infect Dis J (United States) 1999; $18: 552-5$.

17. Roland W.E., McDonald G., Caldwell C.W., Everett E.D. Ehrlichiosis-a cause of prolonged fever. Clin Infect Dis 1995; $20: 821-5$

18. Talbot T.R., Comer J.A., Bloch K.C. Ehrlichia chaffeensis infections among HIV-infected patients in a human monocytic ehrlichiosis-endemic area. Emerg Infect Dis 2003; 9:1123-7.

19. Brouqui P., Raoult D. Endocarditis due to rare and fastidious bacteria. Clin Microbiol Rev 2001;14:177-207.

20. Brouqui P., Raoult D., Durand J.M. Ehrlichia species as possible causative agents of blood culture negative endocarditis. Clin Infect Dis 1995; $1: 148-50$.

21. McGill S.L., Regnery R.L., Karem K.L. Characterization of human immunoglobulin (Ig) isotype and IgG subclass response to Bartonella henselae infection. Infect Immun 1998; 66:5915-20.

22. Ravyn M.D., Goodman J.L., Kodner C.B., et al. Immunodiagnosis of human granulocytic ehrlichiosis by using culture-derived human isolates. J Clin Microbiol 1998;36:1480-8. 